\title{
Unified description for hopping transport in organic semiconductors including both energetic disorder and polaronic contributions
}

\author{
I. I. Fishchuk, ${ }^{1}$ A. Kadashchuk, ${ }^{2,3}$ S. T. Hoffmann, ${ }^{4}$ S. Athanasopoulos, ${ }^{5}$ J. Genoe, ${ }^{2}$ H. Bässler, ${ }^{4}$ and A. Köhler ${ }^{4}$ \\ ${ }^{1}$ Institute for Nuclear Research, National Academy of Sciences of Ukraine, Prospect Nauky 47, 03680 Kyiv, Ukraine \\ ${ }^{2}$ IMEC, Kapeldreef 75, B-3001 Leuven, Belgium \\ ${ }^{3}$ Institute of Physics, National Academy of Sciences of Ukraine, Prospect Nauky 46, 03028 Kyiv, Ukraine \\ ${ }^{4}$ Experimental Physics II and Bayreuth Institute of Macromolecular Research (BIMF), Universitätsstrasse 30, 95448 Bayreuth, Germany \\ ${ }^{5}$ Cavendish Laboratory, University of Cambridge, JJ Thompson Road, Cambridge CB3 OHE, United Kingdom
}

(Received 1 May 2013; revised manuscript received 9 August 2013; published 4 September 2013)

\begin{abstract}
We developed an analytical model to describe hopping transport in organic semiconductors including both energetic disorder and polaronic contributions due to geometric relaxation. The model is based on a Marcus jump rate in terms of the small-polaron concept with a Gaussian energetic disorder, and it is premised upon a generalized effective medium approach yet avoids shortcomings involved in the effective transport energy or percolation concepts. It is superior to our previous treatment [Phys. Rev. B 76, 045210 (2007)] since it is applicable at arbitrary polaron activation energy $E_{a}$ with respect to the energy disorder parameter $\sigma$. It can be adapted to describe both charge-carrier mobility and triplet exciton diffusion. The model is compared with results from Monte Carlo simulations. We show (i) that the activation energy of the thermally activated hopping transport can be decoupled into disorder and polaron contributions whose relative weight depend nonlinearly on the $\sigma / E_{a}$ ratio, and (ii) that the choice of the density of occupied and empty states considered in configurational averaging has a profound effect on the results of calculations of the Marcus hopping transport. The $\sigma / E_{a}$ ratio governs also the carrier-concentration dependence of the charge-carrier mobility in the large-carrier-concentration transport regime as realized in organic field-effect transistors. The carrier-concentration dependence becomes considerably weaker when the polaron energy increases relative to the disorder energy, indicating the absence of universality. This model bridges a gap between disorder and polaron hopping concepts.
\end{abstract}

DOI: 10.1103/PhysRevB.88.125202

PACS number(s): 72.80.Le, 72.20.Ee, 72.80.Ng, 61.43.Bn

\section{INTRODUCTION}

Hopping transport of charge carriers and neutral excitons in disordered organic materials can, in principle, be controlled by both disorder and polaron effects depending on their relative weight. $^{1,2}$ Polaron formation occurs upon adding or removing an electron from a molecule, so the molecular skeleton changes because of the readjustment of the individual bond lengths. The hopping quasiparticle (polaron) is thus a charge associated with a molecular distortion. The change in molecular configuration can also occur upon exciton transfer in an organic solid resulting in the formation of a "polaron exciton." Polaron formation in organic materials is usually treated in terms of the small-polaron model suggested by Holstein ${ }^{3}$ and Friedman $^{4}$ and further developed for nonadiabatic polaron transfer between sites with different energies by Emin. ${ }^{5}$ At high enough temperatures, the small polaron model gives a jump rate equivalent to a Marcus expression. ${ }^{6,7}$

In conventional disordered organic semiconductors chargecarrier transport is dominated by disorder, ${ }^{2,8-12}$ while polaroncontrolled transport prevails in some systems for triplet excitons $^{13,14}$ that feature a narrower density-of-states (DOS) distribution due to their nonpolar nature and consequently a weak interaction with external polarization. The Gaussian disorder model (GDM) $)^{8,9,11}$ has been the most widely used formalism to describe charge-carrier mobility in amorphous organic materials. The model is premised on the argument that charge-carrier transport occurs by hopping through a Gaussian DOS distribution of energetic width $\sigma$. There were several important improvements of the initial GDM suggested by Bässler. ${ }^{8}$ First, spatial correlations of the energies of transport sites in disordered media has been taken into account in order to properly describe the field dependence of the charge mobility. ${ }^{15}$ Another prominent recent advancement was accounting for the partial DOS filling at a large-carrier concentration to describe the carrier-concentration effect on the charge-carrier mobility. The latter led to developing a socalled extended Gaussian disorder (EGD) model ${ }^{16-19}$ which is now conventionally applied to describe charge-carrier mobility in organic field-effect transistors (OFET) and light emitting diodes (OLEDs) in which the current is space-charge limited.

Disorder models are conventionally based on a MillerAbrahams jump rate ${ }^{20}$ and thus they neglect any polaron effects. While considerable efforts have been made for the last two decades in developing the disorder formalism, an adequate description of the charge-carrier transport in disordered organic materials that includes the presence of polaron effects is not yet fully elaborated. In principle, polaron formation can be incorporated in the disorder formalism by using a polaron jump rate model. Some 20 years ago Bässler, Borsenberger, and Perry ${ }^{21}$ suggested that the activation energy of the charge transport in a system with superimposed disorder and polaron effects can heuristically be parametrized by splitting the activation energy into a disorder and a polaron term. Later on, Parris et al. ${ }^{22}$ used the Marcus rate model in Monte Carlo simulations of the charge transport in a disordered organic solid and demonstrated that polaronic carriers with moderate polaron binding energy are consistent with experimental observations. They found that the temperature dependence of the charge mobility in the zero field limit can indeed be 
decoupled into a polaron and a disorder induced contribution as

$$
\mu(T) \propto \exp \left[-\frac{E_{a}}{k_{B} T}-C\left(\frac{\sigma}{k_{B} T}\right)^{2}\right],
$$

where $E_{a}$ is the (small) polaron activation energy, which is equal to half of the polaron binding energy $\left(E_{a}=E_{p} / 2\right), \sigma$ is the width of the DOS distribution, and the constant $C$ weighs the relative contribution of disorder and polaron effects. Parris et al. ${ }^{22}$ deduced $C=0.31$ for $\sigma=80 \mathrm{meV}$ and $E_{a}=75 \mathrm{meV}$. Fishchuk et al. ${ }^{23}$ applied his effective medium approximation (EMA) theory to consider polaron transport in the limiting case of strong polaron effects relative to the disorder contribution $\left(E_{a}>\sigma\right)$ using the so-called symmetrical jump rate, in which the quadratic term in the Marcus expression is neglected, and obtained $C=1 / 8$. This would imply that factor $C$ could not be a constant but depends on the $\sigma / E_{a}$ ratio. In the present paper we address this problem for Marcus hopping using Monte Carlo simulations and EMA analytic calculations at an arbitrary $\sigma / E_{a}$ ratio.

Recently Cottaar et al. ${ }^{24}$ compared Marcus and MillerAbrahams hopping mobilities calculated using a percolationtype theory and found that carrier-concentration dependence of the mobility $\mu(n / N)$, where $n / N$ is carrier concentration per site, is invariant with the strength of polaron effects, i.e., it is universal for an arbitrary $\sigma / E_{a}$ ratio. This contradicts the previous EMA calculations ${ }^{19}$ that predicted a $\mu(n / N)$ dependence to be much weaker for large polaron binding energies as compared to that for small polaron binding energies and to the dependencies typically observed experimentally in conventional organic semiconductors with disorder-controlled charge-carrier transport. ${ }^{17}$

In the present paper we develop a unified model based on a Marcus jump rate model and Gaussian disorder to bridge a gap between disorder-controlled and polaron-controlled transport descriptions that avoids the approximations inherently made in transport models using the effective transport energy or percolation. The model will be compared against results from Monte Carlo simulations. We show that thermally activated hopping transport can indeed be decoupled into a disorder and a polaron contribution but their relative weights depend nonlinearly on the $\sigma / E_{a}$ ratio. We will also show that the $\sigma / E_{a}$ ratio governs the dependence of the charge-carrier mobility on the carrier concentration. Therefore, there is no universality regarding the trade-off between disorder and polaron effects.

Apart from the fundamental aspects the current work is important for analyzing experimental results. Whether transport data is interpreted in terms of a disorder model or a polaron model significantly changes the conclusions drawn about what affects charge-carrier mobility. The usual way to distinguish between polaron and disorder effects is to measure the temperature dependence of the charge-carrier mobility. $\mathrm{A} \ln (\mu) \propto T^{-2}$ dependence is taken as a signature of disorder-controlled transport, while a conventional Arrhenius type of behavior is indicative of polaron transport. However, in view the usually quite limited temperature range, such a discrimination can be ambiguous. In this case the distinction has to rely on a physically meaningful set of transport parameters and a conceptually correct assessment of the interplay of disorder and polaron effects.

\section{MONTE CARLO SIMULATIONS}

Monte Carlo (MC) simulations have been carried out for excitations hopping on a three-dimensional (3D) rectangular lattice. The excitation can, in principle, be equally well a charge carrier or a triplet exciton since the transfer of a triplet can be described as a correlated exchange of two charges. ${ }^{25}$ The physical meaning of the excitation depends on the value chosen for the energetic disorder and the reorganization energy. The advantage in adopting parameters describing a triplet exciton is that the results of the MC simulations can be compared and verified against data available from phosphorescence measurements on poly-( $p$-phenylene) (PPP) type polymer chains, and for this reason we have used parameters compatible with the description of a triplet exciton. ${ }^{14,26}$ The lattice for the simulation was chosen with a spacing of $1.68 \mathrm{~nm}$ between lattice points in the $z$ direction and with a spacing of $1.5 \mathrm{~nm}$ between lattice points in the $x$ and $y$ directions. $1.68 \mathrm{~nm}$ corresponds to the center-to-center distance between two repeat units, and $1.5 \mathrm{~nm}$ is a typical interchain distance. The direction of a polymer chain is thus identified with the $z$ direction. The anisotropy inherent to a polymer chain can be included by using asymmetric coupling strengths described by different inverse localization lengths, i.e., $\gamma_{\|}$for hopping in the $z$ direction and $\gamma_{\perp}$ for hopping in the $x$ or $y$ directions. In contrast, isotropic transport results when choosing identical values for the inverse localization lengths. Energetic disorder is incorporated in the lattice by assigning each site an energy drawn at random from a Gaussian distribution of width $\sigma$ centered around $0 \mathrm{eV}$. In this simulation we have not taken any measures to explicitly include the effect of conjugation length or of correlated disorder.

For one trial in the simulation, an excitation is placed at a site $i$ randomly chosen at the time $t=0$. The excitation thus has a random energy out of the Gaussian distribution. At each Monte Carlo step, the excitation may either hop to a neighboring site $j$ or it may decay. Either of the two events is associated with a certain waiting (dwell) time. For the case of a hop from site $i$ to $j$, the dwell time is given by $\tau_{i j}=-k_{i j}^{-1} \ln (X)$, where $X$ is a random number from a box distribution between 0 and 1 , and $k_{i j}$ is the hopping rate. The dwell time for the decay of a triplet exciton is given by $\tau_{\text {decay }}=-\tau_{\mathrm{ex}} \ln (X)$, with $\tau_{\mathrm{ex}}$ being the lifetime of the triplet exciton. At each MC step, the two dwell times are calculated and the event with the shorter time is chosen. If the chosen event is the decay, the trial is terminated and subsequently a new trial is started with a new exciton at a random site. Monitoring the spatial positions of the initial and final site the exciton has visited, we can deduct values for the effective, mean diffusion coefficient $D=\Delta x^{2} / \Delta t$. A few thousand trials are averaged to obtain meaningful values.

The hopping process depends critically on the rate chosen. In this work we have used a Marcus-type rate given by

$k_{i j}=\frac{J_{i j}^{2}}{\hbar} \sqrt{\frac{\pi}{4 E_{a} k_{B} T}} \exp \left[-\frac{E_{a}}{k_{B} T}-\frac{\varepsilon_{i}-\varepsilon_{j}}{2 k_{B} T}-\frac{\left(\varepsilon_{i}-\varepsilon_{j}\right)^{2}}{16 E_{a} k_{B} T}\right]$,

with $J_{i j}=J_{0} \exp \left(-2 \gamma r_{i j}\right)$. Energy $E_{a}$ is related to the reorganization energy $\lambda$ by $E_{a}=\lambda / 4,{ }^{25} \gamma$ is the inverse 
localization radius related to the electronic coupling matrix element between adjacent sites, $r_{i j}$ is the distance between two sites $i$ and $j$, and $J_{0}$ is a prefactor in the transfer integral. Using the EMA approach, Fishchuk et al. ${ }^{23}$ could show that the discrete rate of Eq. (2) results in the macroscopic equivalent mobility given by Eq. (1) with $C=1 / 8$.

\section{THEORETICAL FORMULATION}

Within the EMA approach the disordered organic medium with localized states for charge carriers is replaced by an effective ordered cubic 3D lattice with spacing $a=N^{-1 / 3}$ equal to the average distance between localized states, where $N$ is the density of the localized states. We consider that energy $\varepsilon$ of the localized states is randomly distributed and their DOS can be described by a Gaussian function

$$
g(\varepsilon)=\frac{N}{\sigma \sqrt{2 \pi}} \exp \left[-\frac{1}{2}\left(\frac{\varepsilon}{\sigma}\right)^{2}\right],
$$

where $\sigma$ is the width of the DOS. Such kinds of the DOS distribution are applicable for both charged and neutral excitations (excitons) in organic disordered solids. ${ }^{2,8}$ It is worth mentioning that since the exchange interactions governs both charge carrier and triplet energy transfer, their diffusion can be described by the same theoretical model as long as the charge-carrier concentration is very low and the electric field acting on charge carriers is sufficiently low so that $e F \Delta x_{i j} \ll$ $\sigma$, where $\Delta x_{i j}$ is the separation of the hopping sites. For the sake of generality, however, we will first formulate a theory of the effective diffusivity of charge carriers at arbitrary carrier concentration and then apply it also to the low concentration limit for both charge carriers and triplet excitations.

For the carrier-concentration $n$ the Fermi energy level $\varepsilon_{F}$ is determined from the transcendental equation

$$
n=\int_{-\infty}^{\infty} d \varepsilon g(\varepsilon) f\left(\varepsilon, \varepsilon_{F}\right),
$$

where $f\left(\varepsilon, \varepsilon_{F}\right)$ is given by the Fermi-Dirac statistics

$$
f\left(\varepsilon, \varepsilon_{F}\right)=\frac{1}{1+\exp \left(\frac{\varepsilon-\varepsilon_{F}}{k_{B} T}\right)} .
$$

In the present work we will apply both the Miller-Abrahams (MA) rates applicable at a weak electron-phonon coupling and/or low temperatures and the Marcus rate model applicable at the strong electron-phonon coupling and/or high temperature limit to describe an elementary hopping transition between two individual sites. For the MA hopping the jump rate $W_{i j}$ for the bare charge carrier between starting $\left(\varepsilon_{i}\right)$ and target $\left(\varepsilon_{j}\right)$ states is given as ${ }^{20}$

$$
\begin{aligned}
W_{i j} & =W_{1} \exp \left[-\frac{\left|\varepsilon_{j}-\varepsilon_{i}\right|+\left(\varepsilon_{j}-\varepsilon_{i}\right)}{2 k_{B} T}\right], \\
W_{1} & =v_{0} \exp \left(-2 \frac{a}{b}\right),
\end{aligned}
$$

where $v_{0}$ is the attempt-to-escape frequency and $b$ is a carrier localization radius. On the other hand, the Marcus hopping model $^{6}$ is applied to account for polaron formation. It describes the polaron hopping with a rate given by Eq. (2), which we rewrite in a form more convenient for further EMA treatment:

$$
\begin{aligned}
& W_{i j}=W_{2} \exp \left[-\frac{\varepsilon_{j}-\varepsilon_{i}}{2 k_{B} T}-\frac{\left(\varepsilon_{j}-\varepsilon_{i}\right)^{2}}{16 E_{a} k_{B} T}\right], \\
& W_{2}=W_{0} \exp \left(-\frac{E_{a}}{k_{B} T}\right),
\end{aligned}
$$

where $E_{a}$ is the is the small polaron activation energy and $W_{0}=\left(J_{0}^{2} / \hbar\right) \sqrt{\pi / 4 E_{a} k_{B} T} \exp (-2 a / b)$.

It is worth nothing that for calculating the effective mobility of charge carrier in disordered media it has already become a common practice to use the concept of so-called effective transport energy. This concept assumes that all carriers jump to target sites of a specific transport energy $E_{\text {transp }}$, which can be calculated by the condition that transition rates to this energy are the highest. An attractive feature of the transport energy approximation is that it effectively reduces the complex hopping transport to a multiple trapping and release formalism. The transport energy $E_{\text {transp }}$ has been calculated for the MA rate in many works ${ }^{11,18,19,27-30}$ and also for a so-called symmetric Marcus-type transition rate. ${ }^{19}$ The latter is an approximate description for the case of high polaron binding energy $E_{a} \gg$ $\left(\varepsilon_{j}-\varepsilon_{i}\right)$, when the quadratic term in the Marcus equation can be neglected. ${ }^{19}$ When using the full Marcus jump rate equation [cf. Eq. (7)] we found that, unfortunately, the calculation of $E_{\text {transp }}$ in this case is problematic if the same mathematical definition for $E_{\text {transp }}$ is used. To the best of our knowledge, no calculation of $E_{\text {transp }}$ for the original Marcus rate has been reported so far.

To circumvent the problem regarding the Marcus hopping, we first pursue an alternative approach for calculating the effective conductivity. This is a key aspect of the present theoretical treatment because this approach does not require calculating $E_{\text {transp }}$ explicitly. Instead, we use direct configurational averaging over a distribution of empty and occupied states of the DOS, which allows us to obtain the effective carrier mobility for both MA and Marcus rates using the Einstein relation at arbitrary polaron activation energies. Since this approach avoids the approximations inherent in the transport energy concepts and the percolation approach, it is more rigorous because it considers not only hopping transitions at a discrete $E_{\text {transp }}$ level. Rather, the whole distribution of unoccupied localizes states, i.e., hopping to all potentially accessible hopping target states, is taken into account. It worth noting that the validity of the Einstein relation in disordered semiconductors under thermal equilibrium condition has been recently confirmed. ${ }^{31}$

In the present work we proceed from calculation of conductivity using an EMA method suggested earlier by Kirkpatrick. ${ }^{32}$ Kirkpatrick has considered the average effects observed in a network of random resistors using an approach of an effective medium. The latter has been defined so that the medium in which the total field inside is equal to the external field. Such a medium must be homogeneous. For simplicity it was assumed to be composed by a set of equal resistors (with conductance $G_{e}$ ) connecting nearest neighbor nodes on the cubic lattice. $G_{e}$ is determined by the following condition - the extra voltages (the local fields), being induced upon replacing $G_{e}$ in this medium with random individual conductances $G_{12}$, should average to zero. ${ }^{32}$ Sequential 
theoretical treatment based on the above condition has resulted in the following relation for the effective conductivity $\sigma_{e}=$ $G_{e} / a$ characterizing the whole disordered system [Eq. (5.4)] in Ref. 8:

$$
\left\langle\frac{\sigma_{12}-\sigma_{e}}{\sigma_{12}+(d-1) \sigma_{e}}\right\rangle=0,
$$

where $\sigma_{12}=G_{12} / a$ is conductivity in two-site cluster approximation, $d$ is dimensionality of the hopping transport system, $G_{12}$ is two-site conductance, and the angular brackets $\langle\cdots\rangle$ denote the configuration averaging. In general, configurational averaging of some value $Q$ is performed by solving a double integral $\langle Q\rangle=\int_{-\infty}^{\infty} d \varepsilon_{1} \int_{-\infty}^{\infty} d \varepsilon_{2} P\left(\varepsilon_{1}\right) P\left(\varepsilon_{2}\right) Q$, where $P\left(\varepsilon_{1}\right)$ and $P\left(\varepsilon_{2}\right)$ denote certain distribution functions for $\varepsilon_{1}$ and $\varepsilon_{2}$, respectively, as detailed below.

The conductance $G_{12}$ can be determined for the MA rate [cf. Eq. (6)] according to Refs. 33 and 34:

$$
G_{12}=G_{1} \frac{\exp \left(-\frac{\left|\varepsilon_{1}-\varepsilon_{2}\right|}{2 k_{B} T}\right)}{4 \cosh \left(\frac{\varepsilon_{1}-\varepsilon_{F}}{2 k_{B} T}\right) \cosh \left(\frac{\varepsilon_{2}-\varepsilon_{F}}{2 k_{B} T}\right)}, \quad G_{1}=\frac{e^{2} W_{1}}{k_{B} T}
$$

and for the Marcus rate [cf. Eq. (7)] as

$$
G_{12}=G_{2} \frac{\exp \left[-\frac{\left(\varepsilon_{1}-\varepsilon_{2}\right)^{2}}{16 E_{a} k_{B} T}\right]}{4 \cosh \left(\frac{\varepsilon_{1}-\varepsilon_{F}}{2 k_{B} T}\right) \cosh \left(\frac{\varepsilon_{2}-\varepsilon_{F}}{2 k_{B} T}\right)}, \quad G_{2}=\frac{e^{2} W_{2}}{k_{B} T} .
$$

To calculate the effective conductivity $\sigma_{e}$ one has to perform a configuration averaging in Eq. (8). It should be pointed out that the appropriate averaging method turns out to be crucial for an adequate description of kinetic characteristics. Site energies $\varepsilon_{1}$ and $\varepsilon_{2}$ enter Eqs. (9) and (10) symmetrically, i.e., $\sigma_{12}=\sigma_{21}$. An elementary method of configurational averaging would be to separately average over starting site $\varepsilon_{1}$ and target site $\varepsilon_{2}$ energies using the product of Gaussian functions $P\left(\varepsilon_{1}\right) P\left(\varepsilon_{2}\right)=g\left(\varepsilon_{1}\right) g\left(\varepsilon_{2}\right)$ given by Eq. (3). Hereafter we shall refer to this averaging method in abbreviation as "averaging B." However, in reality, a charge-carrier jump occurs from an occupied hopping site with energy $\varepsilon_{1}$ to an empty site with energy $\varepsilon_{2}$, and vice versa. Therefore, it is more appropriate to average over the occupied density-of-states (ODOS) distribution for starting site energies and over the unoccupied density-of-states (UDOS) distributions for the target site energies. The localized states occupied by carriers are described by the ODOS distribution $P\left(\varepsilon_{1}\right)$ normalized to unity:

$$
P\left(\varepsilon_{1}\right)=\frac{g\left(\varepsilon_{1}\right) f\left(\varepsilon_{1}, \varepsilon_{F}\right)}{\int_{-\infty}^{\infty} g(\varepsilon) f\left(\varepsilon, \varepsilon_{F}\right) d \varepsilon} .
$$

The empty localized states are described by UDOS distribution $P\left(\varepsilon_{2}\right)$ which in normalized to unity form is given as

$$
P\left(\varepsilon_{2}\right)=\frac{g\left(\varepsilon_{2}\right)\left[1-f\left(\varepsilon_{2}, \varepsilon_{F}\right)\right]}{\int_{-\infty}^{\infty} g(\varepsilon)\left[1-f\left(\varepsilon, \varepsilon_{F}\right)\right] d \varepsilon} .
$$

Let us call this "ODOS-UDOS" averaging method as "averaging $A$ " for short. In the limiting case of vanishing carrier concentration the above relations reduce to $P\left(\varepsilon_{1}\right)=(1 / \sigma \sqrt{2 \pi}) \exp \left[-\left(1 / 2 \sigma^{2}\right)\left(\varepsilon_{1}-\varepsilon_{0}\right)^{2}\right]$ and $P\left(\varepsilon_{2}\right)=$ $(1 / \sigma \sqrt{2 \pi}) \exp \left[-\left(1 / 2 \sigma^{2}\right)\left(\varepsilon_{2}\right)^{2}\right]$, where $\varepsilon_{0}=-\sigma^{2} / k_{B} T$. We will apply first the averaging $A$ method. Substituting Eqs. (11) and (12) into Eq. (8) leads to the following transcendental equation for $\sigma_{e}$ in the case of the MA hopping conductance [Eq. (9)]:

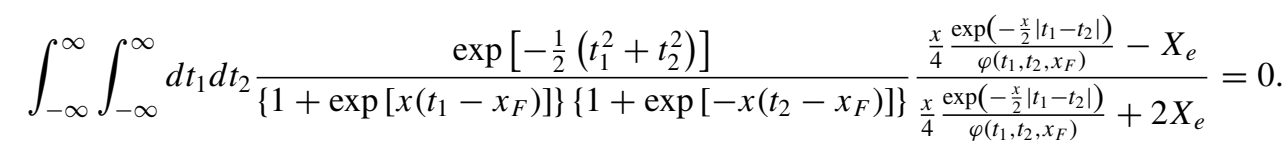

Using the Marcus rate for the hopping conductance [Eq. (10)] it becomes

$$
\int_{-\infty}^{\infty} \int_{-\infty}^{\infty} d t_{1} d t_{2} \frac{\exp \left[-\frac{1}{2}\left(t_{1}^{2}+t_{2}^{2}\right)\right]}{\left\{1+\exp \left[x\left(t_{1}-x_{F}\right)\right]\right\}\left\{1+\exp \left[-x\left(t_{2}-x_{F}\right)\right]\right\}} \frac{\frac{x}{4} \frac{\exp \left[-\frac{x\left(t_{1}-t_{2}\right)^{2}}{16 x_{a}}\right]}{\varphi\left(t_{1}, t_{2}, x_{F}\right)}-Y_{e}}{\frac{x}{4} \frac{\exp \left[-\frac{x\left(t_{1}-t_{2}\right)^{2}}{16 x_{a}}\right]}{\varphi\left(t_{1}, t_{2}, x_{F}\right)}+2 Y_{e}}=0 .
$$

Here $\quad X_{e}=\sigma_{e} / \sigma_{1}, \quad \sigma_{1}=e^{2} W_{1} / a \sigma, \quad Y_{e}=\sigma_{e} / \sigma_{2}, \quad \sigma_{2}=$ $e^{2} W_{2} / a \sigma, \quad x=\sigma / k_{B} T, \quad x_{a}=E_{a} / \sigma, \quad x_{F}=\varepsilon_{F} / \sigma, \quad$ and $\varphi\left(t_{1}, t_{2}, x_{F}\right)=\cosh \left[(x / 2)\left(t_{1}-x_{F}\right)\right] \cosh \left[(x / 2)\left(t_{2}-x_{F}\right)\right]$. The effective mobility $\mu_{e}$ and effective diffusivity $D_{e}$ can be obtained as

$$
\mu_{e}=\frac{\sigma_{e}}{e n}, \quad D_{e}=\frac{k_{B} T}{e} \mu_{e}
$$

where $n$ is the charge-carrier concentration given by Eq. (4).

Let us consider first the charge-carrier transport in the limiting case of very low carrier concentration when carrier concentration per site $n / N \rightarrow 0$. Oelerich et al. ${ }^{35}$ have shown that in this case Eq. (4) yields

$$
\varepsilon_{F}=-\frac{1}{2} \frac{\sigma^{2}}{k_{B} T}+k_{B} T \ln \left(\frac{n}{N}\right) .
$$

It should be noted that the first term in the right side of Eq. (16) should not be confused with the conventional relation describing the thermal quasiequilibrium energy level, as this arises here as a consequence of a Gaussian-shaped DOS and plays a minor role since the second term dominates Eq. (16). At vanishing carrier-concentration $\varepsilon_{F} / \sigma \rightarrow-\infty$. Therefore, hopping transitions from the Fermi level in a Gaussian DOS are eliminated in the low-carrier-concentration transport regime. Instead, the hopping transport is dominated by transitions from the quasiequilibrium energy level $\varepsilon_{\infty}=-\sigma^{2} / k_{B} T$ at 
which, on average, charge carriers migrating within a Gaussian DOS distribution settle in the limit of very weak carrier density.
Substituting Eqs. (15) and (16) into Eq. (13) one obtains a transcendental equation for the effective diffusivity $D_{e}$ in the low-carrier-concentration limit in the case of MA rate:

$$
\int_{-\infty}^{\infty} \int_{-\infty}^{\infty} d t_{1} d t_{2} \exp \left[-\frac{\left(t_{1}+x\right)^{2}+t_{2}^{2}}{2}\right] \frac{\exp \left[-\frac{x}{2}\left|t_{1}-t_{2}\right|-\frac{x}{2}\left(t_{1}+t_{2}\right)-\frac{1}{2} x^{2}\right]-M_{e}}{\exp \left[-\frac{x}{2}\left|t_{1}-t_{2}\right|-\frac{x}{2}\left(t_{1}+t_{2}\right)-\frac{1}{2} x^{2}\right]+(d-1) M_{e}}=0 .
$$

For the Marcus rate the effective diffusivity can be obtained by substituting Eqs. (15) and (16) into Eq. (14) and it reads

$$
\int_{-\infty}^{\infty} \int_{-\infty}^{\infty} d t_{1} d t_{2} \exp \left[-\frac{\left(t_{1}+x\right)^{2}+t_{2}^{2}}{2}\right] \frac{\exp \left[-x x_{a}-\frac{x}{16 x_{a}}\left(t_{1}-t_{2}\right)^{2}-\frac{x}{2}\left(t_{1}+t_{2}\right)-\frac{1}{2} x^{2}\right]-N_{e}}{\exp \left[-x x_{a}-\frac{x}{16 x_{a}}\left(t_{1}-t_{2}\right)^{2}-\frac{x}{2}\left(t_{1}+t_{2}\right)-\frac{1}{2} x^{2}\right]+(d-1) N_{e}}=0
$$

where $M_{e}=D_{e} / D_{1}, N_{e}=D_{e} / D_{2}, D_{1}=a^{2} W_{1}$, and $D_{2}=a^{2} W_{0}$. Note that the Fermi level does not enter in Eqs. (17) and (18), in contrast to Eqs. (13) and (14) derived for the diffusivity in the high-carrier-concentration limit. We should recall that Eqs. (17) and (18) can be used for calculating the effective diffusivity $D_{e}$ of both charge carriers and triplet excitations.

As pointed out above, there could also be another averaging method-averaging $B$. Using this method one obtains instead of Eqs. (13) and (17) the following transcendental equations for the MA rate for arbitrary carrier concentration:

$$
\int_{-\infty}^{\infty} \int_{-\infty}^{\infty} d t_{1} d t_{2} \exp \left[-\frac{1}{2}\left(t_{1}^{2}+t_{2}^{2}\right)\right] \frac{\frac{x}{4} \frac{\exp \left(-\frac{x}{2}\left|t_{1}-t_{2}\right|\right)}{\varphi\left(t_{1}, t_{2}, x_{F}\right)}-X_{e}}{\frac{x}{4} \frac{\exp \left(-\frac{x}{2}\left|t_{1}-t_{2}\right|\right)}{\varphi\left(t_{1}, t_{2}, x_{F}\right)}+2 X_{e}}=0
$$

At vanishing carrier concentration it reduces to

$$
\int_{-\infty}^{\infty} \int_{-\infty}^{\infty} d t_{1} d t_{2} \exp \left[-\frac{1}{2}\left(t_{1}^{2}+t_{2}^{2}\right)\right] \frac{\exp \left[-\frac{x}{2}\left|t_{1}-t_{2}\right|-\frac{x}{2}\left(t_{1}+t_{2}\right)-\frac{1}{2} x^{2}\right]-M_{e}}{\exp \left[-\frac{x}{2}\left|t_{1}-t_{2}\right|-\frac{x}{2}\left(t_{1}+t_{2}\right)-\frac{1}{2} x^{2}\right]+(d-1) M_{e}}=0 .
$$

When using the case of the Marcus rate, the averaging $B$ method yields instead of Eqs. (14) and (18) the following relation:

$$
\int_{-\infty}^{\infty} \int_{-\infty}^{\infty} d t_{1} d t_{2} \exp \left[-\frac{1}{2}\left(t_{1}^{2}+t_{2}^{2}\right)\right]_{\frac{x}{4} \frac{\left.\frac{x}{4} \frac{\exp \left[-\frac{x\left(t_{1}-t_{2}\right)^{2}}{16 x_{a}}\right]}{\varphi\left(t_{1}, t_{2}, x_{F}\right)}-\frac{x\left(t_{1}-t_{2}\right)^{2}}{16 x_{a}}\right]}{\varphi\left(t_{1}, t_{2}, x_{F}\right)}+2 Y_{e}}^{e}=0 .
$$

For vanishing carrier concentration it becomes

$$
\int_{-\infty}^{\infty} \int_{-\infty}^{\infty} d t_{1} d t_{2} \exp \left[-\frac{1}{2}\left(t_{1}^{2}+t_{2}^{2}\right)\right] \frac{\exp \left[-x x_{a}-\frac{x}{16 x_{a}}\left(t_{1}-t_{2}\right)^{2}-\frac{x}{2}\left(t_{1}+t_{2}\right)-\frac{1}{2} x^{2}\right]-N_{e}}{\exp \left[-x x_{a}-\frac{x}{16 x_{a}}\left(t_{1}-t_{2}\right)^{2}-\frac{x}{2}\left(t_{1}+t_{2}\right)-\frac{1}{2} x^{2}\right]+(d-1) N_{e}}=0
$$

As it will be shown below, this averaging $B$ method lead to results obtained in Ref. 24 in which the Marcus rate has been used.

\section{RESULTS OF ANALYTIC CALCULATIONS}

\section{A. The temperature dependence of the charge mobility} at vanishing carrier concentration

Temperature dependencies of the diffusion coefficient $D_{e} / D_{1}$ calculated by Eq. (17) in the low-carrier-concentration limit using both averaging methods $A$ and $B$ [Eq. (20)] are

$$
D_{e}=D_{1} \exp \left(-0.43 x^{2}\right) \quad(\text { averaging A) }
$$

and

$$
D_{e}=D_{1} \exp \left(-0.53 x^{2}\right) \quad \text { (averaging B) }
$$

and are shown in Fig. 1 (curves 1 and 2). Equations (23) and (24) are functionally the same but differ regarding the numerical factor in the exponent. It turns out that only Eq. (23) is consistent with literature work. ${ }^{8,9}$
In the case of Marcus hopping the total activation energy of the effective diffusivity $D_{e}$ is determined by the sum of the

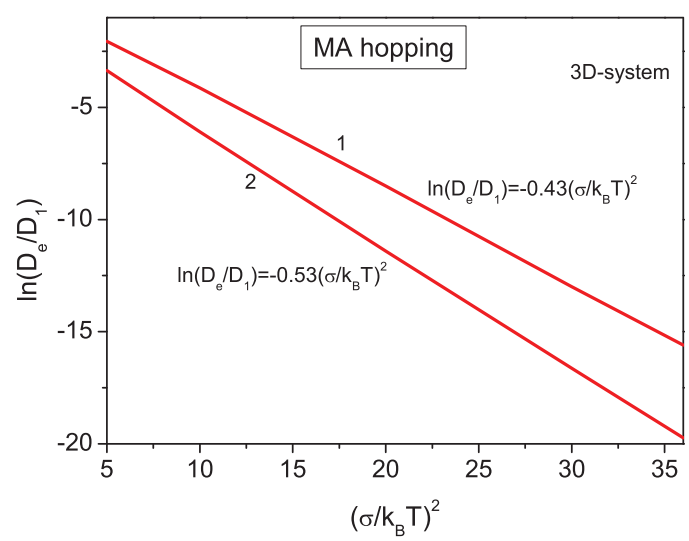

FIG. 1. (Color online) Diffusion coefficient $D_{e} / D_{1}$ as a function of temperature in a $3 \mathrm{D}$ disordered medium at vanishing carrier concentration calculated for the Miller-Abrahams rate by Eqs. (17) and (20) using averaging $A$ and $B$ (curve 1 and 2, respectively). 


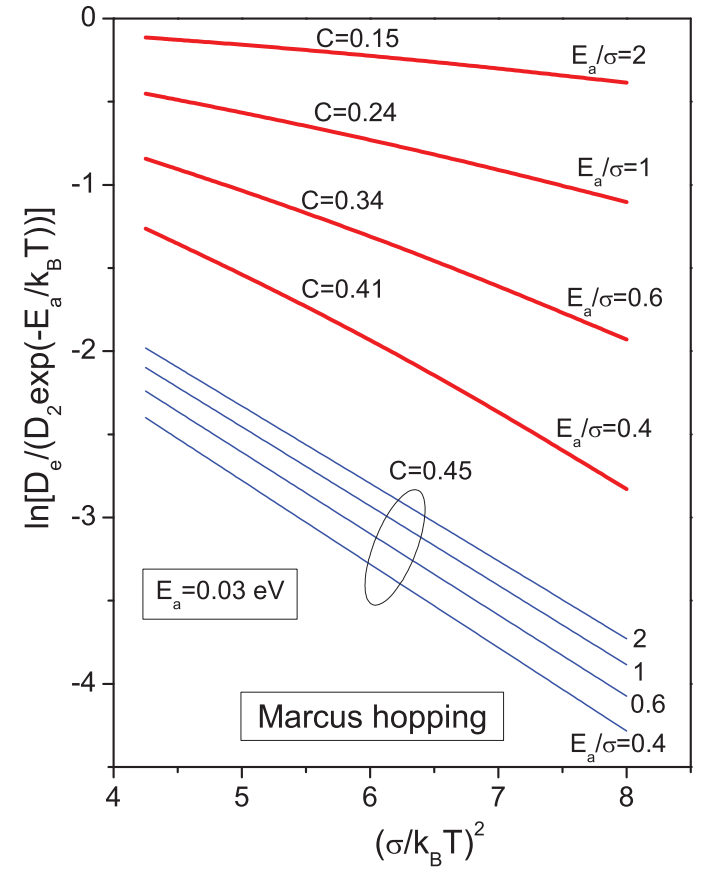

FIG. 2. (Color online) The $D_{e} /\left[D_{2} \exp \left(-E_{a} / k_{B} T\right)\right]$ vs $\left(\sigma / k_{B} T\right)^{2}$ calculated for Marcus hopping in a $3 \mathrm{D}$ system at vanishing carrier concentration by Eq. (16) using averaging $A$ (red solid curves) and by Eq. (22) using averaging $B$ (blue thin curves) for different $E_{a} / \sigma$ ratios at $E_{a}=0.03 \mathrm{eV}$. Factor $C$ denotes the slope of the temperature dependencies.

polaron activation energy $E_{a}$ and a contribution due to energetic disorder [cf. Eq. (1)]. The energy disorder contribution can be evaluated as $D_{e} /\left[D_{2} \exp \left(-E_{a} / k_{B} T\right)\right]$. Figure 2 shows the temperature dependence of $D_{e} /\left[D_{2} \exp \left(-E_{a} / k_{B} T\right)\right]$ calculated for the Marcus rate in a 3D system by Eq. (16) using averaging $A$ (red bold curves) and by Eq. (22) using averaging $B$ (blue thin curves) at a polaron activation energy $E_{a}=$ $0.03 \mathrm{eV}$ and different $E_{a} / \sigma$ ratios. It is evident from Fig. 2 that the temperature dependencies calculated for the Marcus rate depend drastically on the method of configurational averaging used. While the averaging $B$ predicts a temperature dependence of $D_{e} /\left[D_{2} \exp \left(-E_{a} / k_{B} T\right)\right]$ independent of the $E_{a} / \sigma$ ratio (Fig. 2, blue thin curves), the averaging $A$ method results in temperature dependence that does depend on $E_{a} / \sigma$ and becomes progressively weaker with increasing $E_{a} / \sigma$ ratio (red curves). Below we will show that only the latter calculation method yields results consistent with Monte Carlo simulation data.

\section{B. Carrier-concentration dependence of the charge mobility}

Now we consider hopping transport at an arbitrary carrier density for the Marcus rate. Figure 3(a) presents the carrierconcentration dependencies of the Marcus hopping mobility $\mu_{e} / \mu_{2}=(N / n) Y_{e} \exp \left(-x x_{a}\right)$ calculated for different temperatures by Eqs. (14) and (15) using the averaging $A$ method both at a large relative polaron activation energy $E_{a} / \sigma=3$ (dashed curves) and for a small $E_{a} / \sigma=0.5$ ratio (solid curves). The calculations clearly show that the effective charge-carrier mobility depends very weakly on the carrier concentration even at low temperatures in the case when

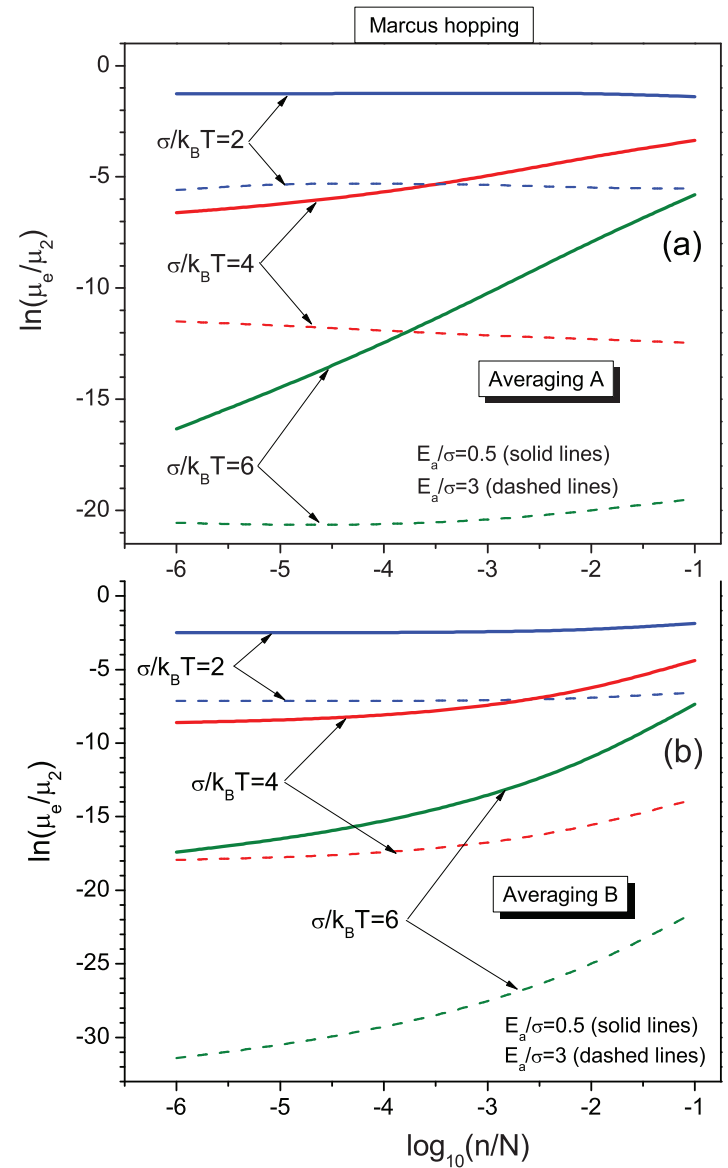

FIG. 3. (Color online) The charge-carrier mobility $\mu_{e} / \mu_{2}=$ $(N / n) Y_{e} \exp \left(-x x_{a}\right)$ vs carrier-concentration $n / N$ calculated for the Marcus rate by Eqs. (14) and (15) using averaging $A$ (a) and by Eqs. (15) and (21) using averaging $B$ (b) for different temperatures at $E_{a} / \sigma=0.5$ (solid curves) and $E_{a} / \sigma=3$ (dashed curves).

polaron effects dominate over the energy disorder effects $\left(E_{a}>\sigma\right)$, while this dependence appears to be strong, especially at low temperatures, when the polaron activation energy is relatively small $\left(E_{a}<\sigma\right)$.

For comparison, the carrier-concentration dependencies of the Marcus hopping mobility calculated using the averaging $B$ method for different temperatures and two different $E_{a} / \sigma$ ratios are presented in Fig. 3(b). Apart from a vertical shift of the calculated dependencies with respect to each other, these dependencies are virtually invariant with $E_{a} / \sigma$ ratio, and at low temperatures they are similarly strong in a system with large $\left(E_{a} / \sigma=3\right)$ and small $\left(E_{a} / \sigma=0.5\right)$ polaron activation energy as depicted in Fig. 3(b) by dashed and solid curves, respectively. Thus, the averaging $B$ method, when applied to the Marcus rate, is independent to the relative weight between polaron and disorder contributions to the charge transport. This is at variance with results obtained by employing the averaging $A$ method [see Fig. 3(a)].

Figure 4 shows the carrier-concentration dependencies of the Marcus hopping mobility calculated by averaging $A$ and $B$ methods at a constant temperature $\left(\sigma / k_{B} T=4\right)$ parametric in $E_{a} / \sigma$ ratios. The results shown in Fig. 4 show clearly that when using the averaging $A$ method the carrier-concentration effect depends strongly on the $E_{a} / \sigma$ ratio. This dependence becomes 


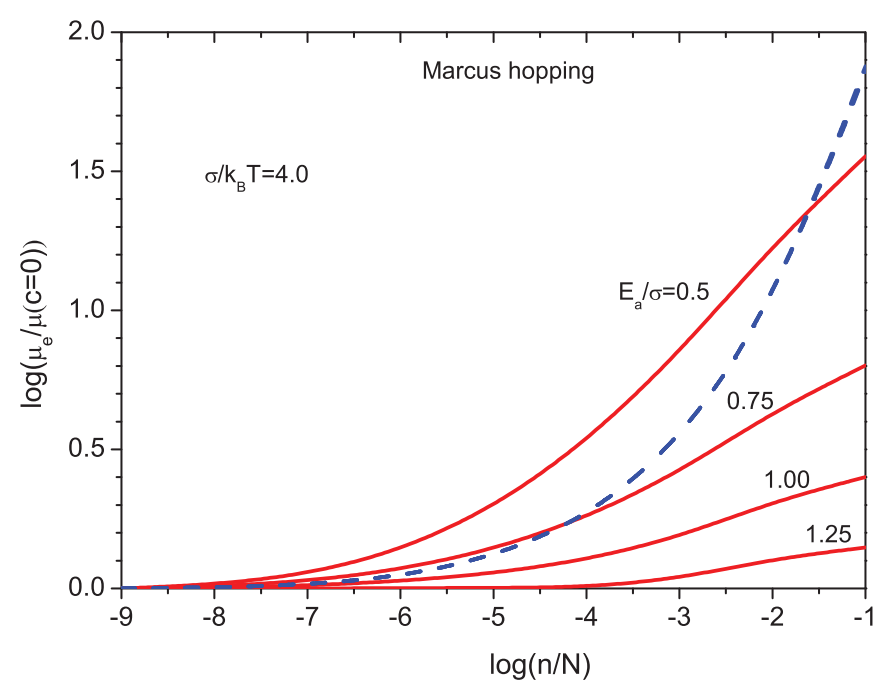

FIG. 4. (Color online) The carrier-concentration dependencies of Marcus hopping mobility normalized to the zero carrier-concentration mobility ( $c=n / N \rightarrow 0$ ) calculated by Eqs. (14), (15), and (21) using averaging $A$ (red solid curves) for $\sigma / k_{B} T=4$ and using averaging $B$ (blue dashed curves) for several $E_{a} / \sigma$ ratios indicated in the figure. Note that all four dashed curves coincide.

weaker as the polaron contribution to the activation energy to transport becomes more important (red solid curves). In the case of averaging $B$ the carrier-concentration dependencies are invariant with $E_{a} / \sigma$ ratio and the calculated curves merge (blue dashed curves).

For completeness sake we compare the averaging methods $A$ and $B$ for calculating the carrier-concentration dependencies of the charge-carrier mobility also for the MA rate. Figure 5 shows the effective mobility $\mu_{e} / \mu_{1}=(N / n) X_{e}$ calculated as a function of carrier concentration for MA rate by Eqs. (13), (15), and (19) using both averaging methods. Evidently that when using the MA hopping rate both averaging $A$ and $B$ methods yield similar dependencies on the charge-carrier concentration (Fig. 5), indicating that the choice between these averaging methods is irrelevant. The latter is in contrast to the

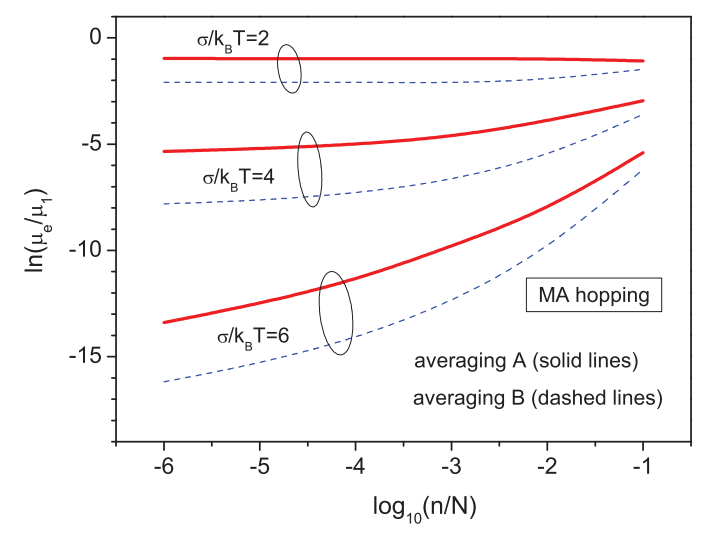

FIG. 5. (Color online) The charge-carrier mobility [derived via conductivity as $\left.\mu_{e} / \mu_{1}=(N / n) X_{e}\right]$ vs carrier-concentration $n / N$ for different temperatures calculated for Miller-Abrahams rate by Eqs. (13), (15), and (19) using averaging $A$ (solid lines) and $B$ (dashed lines). case of the Marcus hopping where the averaging method does matter [cf. Figs. 3(a) and 3(b)].

\section{Comparison with computer simulation results}

As demonstrated in the preceding section, the calculation of the charge-carrier mobility for the Marcus rate is very sensitive to the averaging method used in Eq. (8). For instance, for $E_{a} / \sigma=2$ the averaging $A$ [cf. Eq. (18)] yields

$$
\mu_{e}=\mu_{2} \exp \left(-x x_{a}-C_{1} x^{2}\right), \quad C_{1}=0.15,
$$

while using the averaging $B$ method [Eq. (22)] one obtains

$$
\mu_{e}=\mu_{2} \exp \left(-x x_{a}-C_{2} x^{2}\right), \quad C_{2}=0.44
$$

Thus, coefficients $C_{1}$ and $C_{2}$ for the same ratio $E_{a} / \sigma=2$ differ considerably for these two methods. To resolve the discrepancy, the present EMA calculations were compared with Monte Carlo (MC) simulations data obtained for the temperature dependent diffusion of triplet excitons. The latter approach could be regarded as a "computer experiment" and it actually allows us to check the validity of approximations in analytical treatments that are based on the same physical principles. The MC simulations themselves had been verified by comparison against phosphorescence data. ${ }^{14}$

Figure 6 shows results of MC simulations of the temperature dependent diffusivity of triplet excitations in a 3D lattice using the Marcus rate [Eq. (2)] at $E_{a}=30 \mathrm{meV}$ and different width of the DOS $\sigma$ over a broad temperature range. The use of the Marcus rate implies that the resulting diffusivity depends on both the polaron activation energy $E_{a}$ and the energetic disorder $\sigma^{2} / k_{B} T$, analogous to the mobility [Eq. (1)]. In order to extract only the disorder contribution, and in particular the weighing factor $C$, we have divided the diffusivity by $\exp \left(-E_{a} / k_{B} T\right)$ and plotted the resulting quantity as a function of $1 / T^{2}$. The $C$ factor can then readily be read off from the slope of the curve in the high-temperature branch. From Fig. 6 it is clearly apparent that the slope is not identical

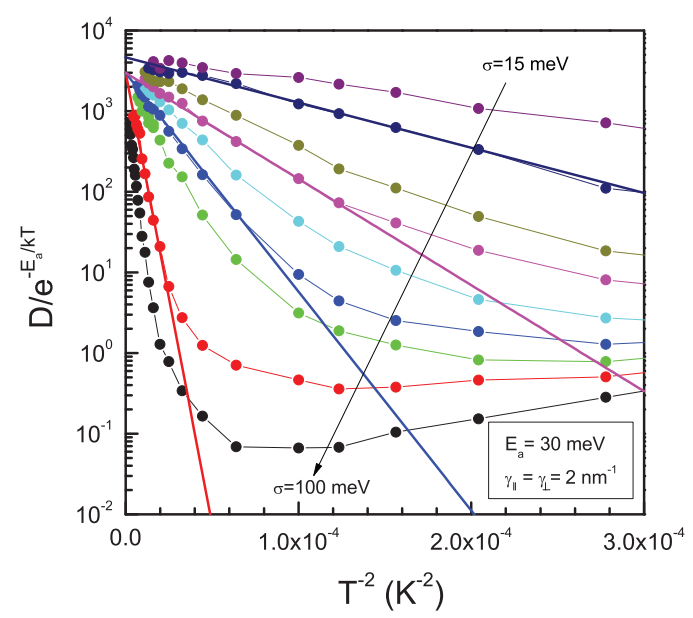

FIG. 6. (Color online) Monte Carlo simulation of temperature dependencies of the coefficient of diffusion $D / \exp \left(-E_{a} / k_{B} T\right)$ of triplet excitons parametric in the width of the DOS which varies as follows: $\sigma=15,20,25,30,35,40,50,70$, and $100 \mathrm{meV}$. Simulations are done for an isotropic 3D lattice using a Marcus rate at $E_{a}=$ $30 \mathrm{meV}$. Four slopes are indicated for illustration. 


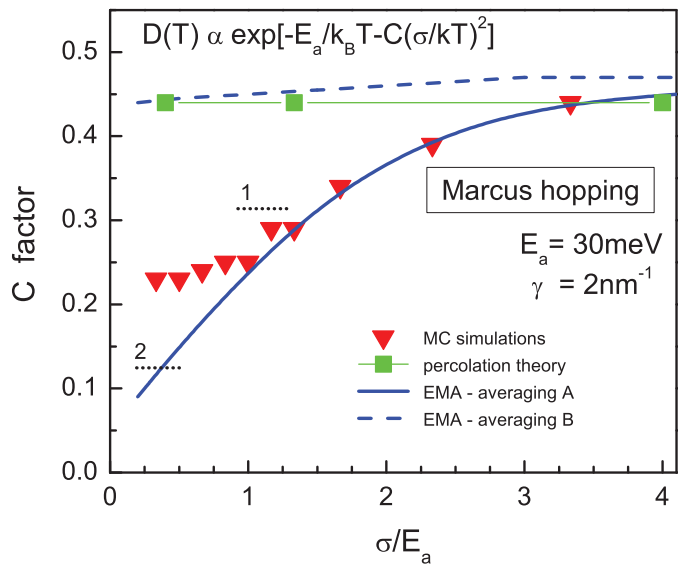

FIG. 7. (Color online) Factor $C$ vs $\sigma / E_{a}$ obtained for Marcus hopping in 3D system by Monte Carlo simulations (triangles); derived from $D_{e}=\left(k_{B} T / e\right) \mu_{e}$ using the mobility calculated by the present EMA theory adopting both configuration averaging $A$ and $B$ for $E_{a}=30 \mathrm{meV}$ (solid and dashed blue curves, respectively), and by the percolation-based scaling theory from Ref. 24 (squares with green connecting line). Short dotted lines 1 and 2 shows results from Refs. 22 and 23, respectively.

for different ratios of $\sigma / E_{a}$. We focus here only on the high-temperature branches of the data which corresponds to the nondispersive transport regime of triplet excitations which are in thermal equilibrium. At low temperatures, transport becomes dispersive featuring different temperature dependencies (Fig. 6). This nonequilibrium transport regime has been discussed in an earlier publication. ${ }^{14}$

Figure 7 shows the factor $C$ derived from the $\mathrm{MC}$ simulations data from Fig. 6 (symbols) for different $\sigma / E_{a}$ ratios as well as the $C$ factor obtained by translating the charge-carrier mobility into diffusivity by using the Einstein relation $D_{e}=\left(k_{B} T / e\right) \mu_{e}$ from the present EMA theory using both configuration averaging $A$ and $B$. The factor $C$ obtained by the MC simulations decreases with decreasing $\sigma / E_{a}$ ratio, i.e., with decreasing the relative strength of the disorder effects. At large $\sigma / E_{a}$ it approaches the predicted value 0.44 as in the case of Miller-Abrahams hopping.

Figure 7 clearly show that the averaging $A$ method provides a reasonably good agreement with $\mathrm{MC}$ simulation data (solid curve), while the results obtained when using the averaging $B$ method are in disagreement with the simulation. Evidently the averaging $A$ method is superior to the averaging $B$ method. In passing we note that the present calculations are in good agreement with the previous EMA calculations ${ }^{19,23}$ performed before for Marcus hopping in the limiting case of large polaron activation energy $E_{a} / \sigma \gg 1$ and using the transport energy concept. The latter corroborates the approach employed in Ref. 19.

Figure 8 presents the factor $C$ vs $\sigma / E_{a}$ for different degree of system anisotropy obtained for Marcus rate by $\mathrm{MC}$ simulations (symbols) and the EMA calculations utilizing the averaging $A$ for two values of the dimensionality $d$ (solid curves). Here to fit the MC data for an anisotropic system, the $d$ was formally taken as a fitting parameter in the EMA calculations.

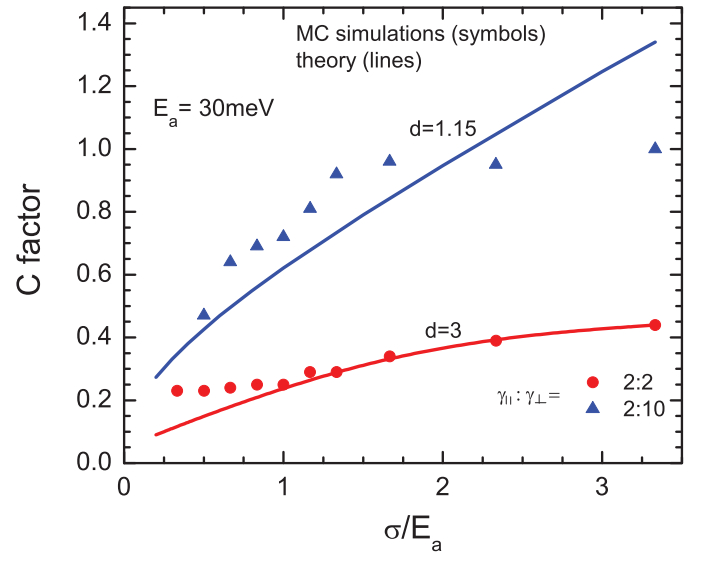

FIG. 8. (Color online) Factor $C$ vs $\sigma / E_{a}$ at different anisotropy of a disordered system obtained for Marcus hopping by Monte Carlo simulations (symbols) and by the present EMA theory by Eq. (18) using configuration averaging $A$ for $E_{a}=30 \mathrm{meV}$ (solid curves). "Dimensionality" $d=1.15$ was just chosen as a fitting parameter to match the simulation data.

The MC simulations show that in a system with anisotropic transport the $C$ factor changes more strongly with the $\sigma / E_{a}$ ratio and approaches $C \approx 1$ at large ratios as expected for $1 \mathrm{D}$ transport. ${ }^{36}$ As one can see from Fig. 8, the present EMA model fits the MC simulation data reasonably well at least at moderate $\sigma / E_{a}$ ratios and correctly predicts the change of the $C\left(\sigma / E_{a}\right)$ dependence in an anisotropic system except for a highly anisotropic system with $\sigma / E_{a}>2$. It is conceivable that under this circumstance one enters the Marcus inversion region.

It is important to compare the above results obtained by the averaging $A$ method [cf. Eq. (25)] with the recent results obtained by Cottaar et al. ${ }^{24}$ who employed several calculations methods including a similar EMA Kirkpatrik's approach ${ }^{32}$ and also the numerical master equation method. Let us consider the data from Table 1 in Ref. 24 for Marcus hopping where the authors fitted their Eq. (5a) to $\mu_{0}(T) \propto \exp \left(-C^{*} x^{2}\right)$ and for values $\sigma / E_{a}=0.4,1.33,4$ they obtained $C^{*}=0.69$, $0.49,0.44$, respectively. Analyzing those data show that if one fits their data to the relation $\mu_{0}(T) / \exp \left(-E_{a} / k_{B} T\right) \propto$ $\exp \left(-C x^{2}\right)$ used in the present EMA theory, then the factor $C=0.44$ is found to be the same for all ratios $\sigma / E_{a}$ (for $0.4,1.33$, and 4) used in Ref. 24. Thus, the calculations in Ref. 24 predict $C=0.44$ invariant with the ratio $\sigma / E_{a}$ (Fig. 7, squares). This is in sharp contrast to both the Monte Carlo simulation data and the present EMA calculations utilizing the averaging $A$ method (triangles and solid line in Fig. 7, respectively).

On the other hand, our calculations yield basically the same results as in Ref. 24 provided that the "averaging $B$ " method is employed [i.e., using the product of Gaussian functions $P\left(\varepsilon_{1}\right) P\left(\varepsilon_{2}\right)=g\left(\varepsilon_{1}\right) g\left(\varepsilon_{2}\right)$ given by Eq. (3)]. The discrepancy arises only for the Marcus mobility in the case when the "averaging $A$ method" is used and this straightforwardly proves that the averaging method is a key cause for the difference observed. In this respect it should be noted that the master equation method usually neglects any correlations between the occupational probabilities of different sites and assumes that a site $i$ is given by its energy which is drawn 
randomly ${ }^{37}$ from a Gaussian distribution with variance $\sigma$. This is basically equivalent to the our averaging $B$ method and therefore it seems to be natural that results of our EMA calculations using such an averaging method coincide with the results of Ref. 24. The fact that the result of Ref. 24 has been recovered using the averaging $B$ method testifies to the applicability of our approach. On the other hand, averaging $A$ implies configurational averaging over ODOS and UDOS distributions for the starting and target states, respectively, and this was never used in the master equation calculations. The fact that a different result is obtained with the averaging $A$ method is evidence that the physics question, i.e., which part of the DOS is considered, is central to the problem.

Finally, a hint for the possible origin of the discrepancy coming from different averaging methods can be obtained from the following simplified analysis which shows that it is not about a technical issue of how to perform an averaging procedure but it is about the physical question of which part of the DOS to consider. Let us assume for simplicity that hopping target states $\varepsilon_{2}$ are located at the maximum of the DOS distribution and consider the vanishing carrier-concentration limit. Furthermore, we assume that energies of the starting hopping states $\varepsilon_{1}$ are given by the DOS and ODOS distribution and calculate integrals of exponents from Eqs. (17), (18), (20), and (22) yielding the "intersite mobility" (left-hand summands in numerators) for the MA and Marcus hopping transitions from the starting DOS and ODOS hopping states to the maximum of the DOS distribution. The quadratic term in the Marcus jump equation can be ignored here at $\sigma / E_{a} \ll 1$. In other words, we calculate the following values:

$$
\begin{aligned}
Z_{\mathrm{DOS}(\mathrm{ODOS})}^{\mathrm{MA}}= & \int_{-\infty}^{\infty} d \varepsilon_{1} g_{\mathrm{DOS}(\mathrm{ODOS})}\left(\varepsilon_{1}\right) \\
& \times \exp \left[-\frac{\left|\varepsilon_{1}\right|+\varepsilon_{1}}{2 k_{B} T}-\frac{1}{2}\left(\frac{\sigma}{k_{B} T}\right)^{2}\right] \\
Z_{\mathrm{DOS}(\mathrm{ODOS})}^{\mathrm{Marcus}}= & \int_{-\infty}^{\infty} d \varepsilon_{1} g_{\mathrm{DOS}(\mathrm{ODOS})}\left(\varepsilon_{1}\right) \\
& \times \exp \left[-\frac{E_{a}}{k_{B} T}-\frac{\varepsilon_{1}}{2 k_{B} T}-\frac{1}{2}\left(\frac{\sigma}{k_{B} T}\right)^{2}\right]
\end{aligned}
$$

Here

$$
\begin{aligned}
g_{\text {DOS }}\left(\varepsilon_{1}\right) & =\frac{1}{\sigma \sqrt{2 \pi}} \exp \left[-\frac{1}{2}\left(\frac{\varepsilon_{1}}{\sigma}\right)^{2}\right], \\
g_{\text {ODOS }}\left(\varepsilon_{1}\right) & =\frac{1}{\sigma \sqrt{2 \pi}} \exp \left[-\frac{1}{2}\left(\frac{\varepsilon_{1}-\varepsilon_{\text {eq }}}{\sigma}\right)^{2}\right], \\
\varepsilon_{\text {eq }} & =-\frac{\sigma^{2}}{k_{B} T} .
\end{aligned}
$$

From Eqs. (27)-(29) for $\sigma / k_{B} T=2.5$ one obtains

$$
\frac{Z_{\mathrm{ODOS}}^{\mathrm{MA}}}{Z_{\mathrm{DOS}}^{\mathrm{MA}}}=1.55, \quad \frac{Z_{\mathrm{ODOS}}^{\mathrm{Marcus}}}{Z_{\mathrm{DOS}}^{\mathrm{Marcus}}}=22.76 .
$$

From the above ratios one can conclude that the intersite mobility integral Eq. (27) changes just slightly (=1.55) for the MA rate when configurational averaging over the ODOS is used instead of the DOS. This fact is also reflected in the calculated effective MA mobility (cf. Fig. 1)-the latter was found to be virtually insensitive to the method of configurational averaging. However, the situation is drastically different in the case of the Marcus rate [see the righthand part of Eq. (30)], where the intersite mobility integral Eq. (28) changes much strongly $(=22.76)$ upon changing the configurational averaging method. This corresponds well to the considerable change of the effective Marcus mobility found above (cf. Fig. 2) resulting in the significant change of the $C$ parameter (Fig. 7), which was also confirmed by the Monte Carlo simulations for the Marcus rates at $\sigma / E_{a} \ll 1$. Thus, the choice of certain averaging method becomes important only in the case of Marcus rates at $E_{a} / \sigma \gg 1$. Therefore, there is no reason to expect a universal behavior with respect to the rate model claimed in Ref. 24.

\section{DISCUSSION AND CONCLUSIONS}

The present results prove that the way of configurational averaging in Eq. (8) for calculating the conductivity for the Marcus hopping regime plays a crucial role in disordered organic solids. While the averaging $A$ method yields the calculated $C$ factor at different $\sigma / E_{a}$ ratios in perfect agreement with MC simulation data, the averaging $B$ method results in a virtually constant $C$ factor (Fig. 7) that is in sharp contrast with the above simulations. This could be used as a critical test for applicability of the calculation method and validates the approach based on averaging $A$. In the latter case, one considers averaging the energies $\varepsilon_{1}$ of starting sites and $\varepsilon_{2}$ for target sites over the ODOS and UDOS distributions, respectively. The normalized ODOS and UDOS distributions with respect to the DOS calculated at large carrier concentration by Eqs. (11) and (12), respectively, are shown in Fig. 9. The calculated Fermi level is also shown

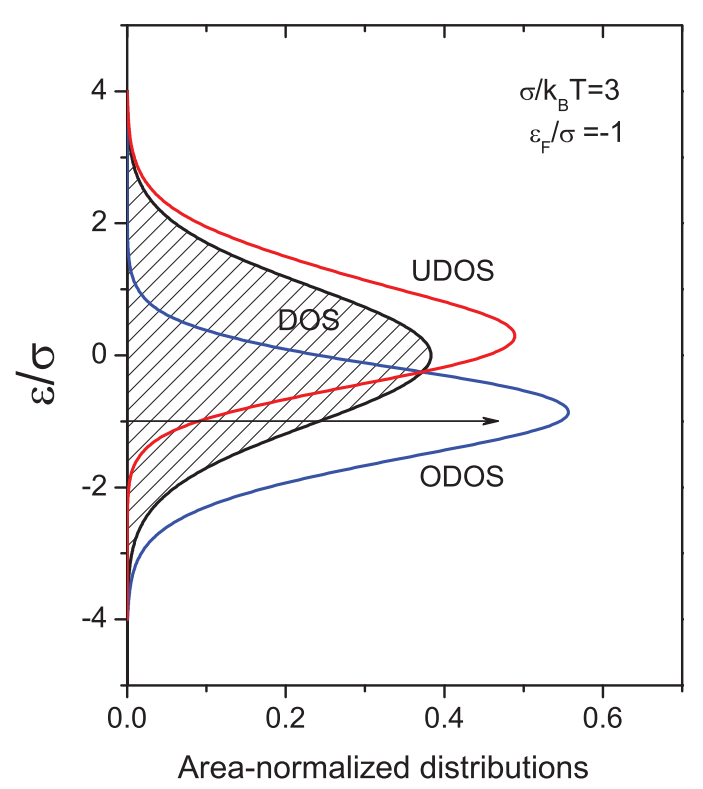

FIG. 9. (Color online) Normalized ODOS (blue line) and UDOS (red line) distributions in a disordered organic system with a Gaussian DOS (patterned curve) calculated by Eqs. (11) and (12), respectively, at large carrier concentration. The arrow indicates the position of the Fermi level calculated by Eq. (5) for the considered carrierconcentration $\varepsilon_{F} / \sigma=-1$ that corresponds to $n / N=1.9 \times 10^{-1}$. Note that all curves are normalized by integral area to unity. 
in Fig. 9 by an arrow. The averaging $A$ method takes into account that at high-carrier concentration carriers can only jump to unoccupied states located in the upper part of the DOS described by $P\left(\varepsilon_{2}\right)$ [Eq. (12)] since the lower states are occupied. At the vanishing carrier-concentration limit, when the Fermi level is settled in deep tail states, hopping transitions to any sites are in principle possible since the DOS is virtually empty, and Eq. (12) becomes equivalent to the DOS distribution $g(\varepsilon)$ [Eq. (3)]. The ODOS distribution [Eq. (11)] is located in lower part of the DOS (Fig. 9) and describes which energy levels a carrier visits in the course of its hopping motion.

A key result of the present combined MC simulations and the analytical calculations studies is that the $C$ factor, which weighs the relative contribution of disorder and polaron effects as represented by the $\sigma / E_{a}$ ratio, is not a constant but significantly decreases with decreasing $\sigma / E_{a}$, i.e., with increasing polaron formation energy in the same disordered system. This agrees with previous studies where $C=0.31$ and $1 / 8$ (cf. Fig. 7, short dotted lines) was obtained in Refs. 22 and 23 , respectively. It is, however, at variance with results reported in Ref. 24, where the authors applied a percolation-based theory and EMA calculations based on the same Kirkpatrick's method $^{32}$ for calculation of the charge-carrier mobility and found the $C$ factor independent on the $\sigma / E_{a}$ ratio. The latter behavior is also shown in our EMA calculations based on the averaging $B$ method which we therefore regard as inappropriate in the case of Marcus hopping. Furthermore, the present EMA calculations based on the averaging $A$ demonstrated that the carrier-concentration dependence of the charge-carrier mobility $\mu(n / N)$ is not universal as claimed in Ref. 24 but also depends on the $\sigma / E_{a}$ ratio (cf. Fig. 4). The present results corroborate a very weak $\mu(n / N)$ dependence $^{19}$ found before in calculations using the symmetrical jump rated model in a system with $\sigma / E_{a}=0.33$.

The above formalism that relates the $C$ factor to the transport parameters $\sigma$ and $E_{a}$ can be used to establish guidelines for the assessment of the relative weight of disorder and geometric distortion effects on electronic transport. Since the formalism is independent of the strength of coupling it is applicable to both charge carriers as well as neutral excitons as long as the electric field acting on charge carriers is low enough so that the drop of the electrostatic potential across the transport sites is much less that the difference of the site energies themselves. Disorder and reorganization effects are comparable when the activation energies that enter the exponents in Eq. (1) are equal. This is defines a critical temperature $T_{c}$,

$$
T_{c}=\frac{C}{k_{B} E_{a}} \sigma^{2} .
$$

For $T>T_{C}$ transport is polaron controlled, while for $T<T_{C}$ it is disorder controlled. Based upon the established correlation between the $C$ factor and the pertinent transport parameters $\sigma$ and $E_{a}$ one can calculate $T_{c}$ as a function of $\sigma$ parametric in the $\sigma / E_{a}$ ratio. If the $C$ factor was constant, $T_{c}$ would vary quadratically with $\sigma$. When inserting into Eq. (27) the calculated dependence of the $C$ factor on $\sigma / E_{a}$ that is displayed in Figs. 7 and 8 for $E_{a}=30 \mathrm{meV}$ for the three-dimensional case, one arrives at an approximate relation $T_{c} \sim \sigma^{2.5}$. This is illustrated in Fig. 10 for different values of

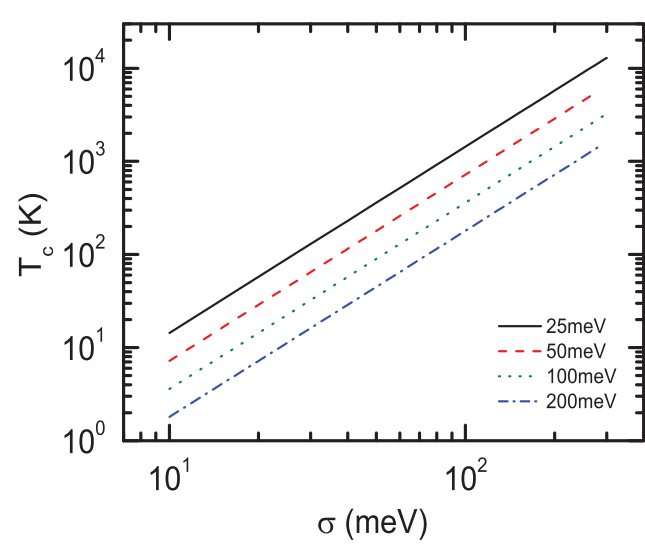

FIG. 10. (Color online) The variation of $T_{c}$ on the disorder parameter $\sigma$ for different values of $E_{a}$ as indicated in the figure.

$E_{a}$. The important message evident from Fig. 10 is that the critical temperature $T_{c}$ scales approximately with $\sigma^{2} / E_{a}$, i.e., it increases superquadratically with the disorder parameter $\sigma$ but only linearly with $1 / E_{a}$. Therefore, the relative disorder contribution increases with increasing (static) disorder.

This phenomenon is borne out by experimental results on the transport of triplet excitons as well as charge carriers. In the case of triplet excitons the disorder parameters are amenable from the inhomogeneous broadening of phosphorescence spectra, and the geometric reorganization can be inferred from their Huang-Rhys factors that determine the relative strength of the vibronic components. An analysis of phosphorescence spectra of a series of $\pi$-conjugated polymers with phenylene as the central building unit in the polymer chain shows that $\sigma$ increases from $29 \mathrm{meV}$ for the weakly disordered methylsubstituted ladder-type poly(para-phenylene) (MeLPPP) to $70 \mathrm{meV}$ for the more strongly disordered alkoxy-substituted poly-phenylene (DOO-PPP), ${ }^{38}$ while the polaron activation energy $E_{a}$ are between $65 \mathrm{meV}$ (MeLPPP) and $81 \mathrm{meV}$ (DOO-PPP). ${ }^{26}$ Using the data from Fig. 7 this yields $C$ factors ranging from 0.125 to 0.2 . Such $C$ factors translate into critical temperatures from $17 \mathrm{~K}$ (MeLPPP) to $140 \mathrm{~K}$ (DOO-PPP). This indicates that, for triplet excitons at moderate temperatures and in particular at room temperature, transport is controlled by geometric reorganization. Disorder effects prevail only at lower temperatures. This is consistent with simulation work. ${ }^{14}$ When estimating those $T_{c}$ temperatures one should be aware, though, the theory developed above rests upon the notion of quasiequilibrium. This is no longer granted at very low temperatures when $\sigma / k_{B} T$ exceeds $3.5-4.0$ and transport becomes dispersive.

The situation is reversed when considering charge transport. The reason is that a triplet excitation is a neutral, localized two particle entity as compared as a single, more spread-out, charge that is highly susceptible to fluctuations in the environmental polarization. ${ }^{25}$ We recently investigated hole transport in a series of $\pi$-conjugated copolymers. ${ }^{39}$ When measuring the temperature dependence of the hole mobility $\sigma$ values have been obtained ranging from 91 to $109 \mathrm{meV}$, quite consistent with many other disordered organic semiconductors ${ }^{2}$ and significantly higher than the $\sigma$ values of triplets. Values for $E_{a}$, inferred from DFT calculations, range between 25 and 
$46 \mathrm{meV},{ }^{39}$ i.e., half that for triplet exciton transport (see above). The corresponding values for the $C$ factor are 0.40 to 0.44 and the $T_{c}$ values are between 1100 and $1300 \mathrm{~K}$. It is obvious that the increased disorder combined with the lower reorganization energy of charge carriers ensures that at an experimentally relevant temperature range charge transport is dominated by disorder, tractable in terms of Miller-Abrahams rate instead of the computationally more demanding Marcus rates.

\section{ACKNOWLEDGMENTS}

The research was supported by the GRK1640 of the DFG, by the Royal Society, UK, through an international exchange grant, by the European Project POLARIC (FP7-247978), by the NAS of Ukraine via the program of fundamental research on nanophysics (Project No.1/13-H-23K), and the UkrainianLithuanian collaboration project M/318-2013 of DKNII of Ukraine.
${ }^{1}$ H. Pope and C. E. Swenberg, Electronic Processes in Organic Crystals, 2nd ed. (Oxford University Press, Oxford, 1999).

${ }^{2}$ H. Bässler and A. Köhler, Top. Curr. Chem. 312, 1 (2012).

${ }^{3}$ T. Holstein, Ann. Phys. 8, 325 (1959).

${ }^{4}$ L. Friedman, Phys. Rev. 135, A233 (1964).

${ }^{5}$ D. Emin, Phys. Rev. Lett. 32, 303 (1974).

${ }^{6}$ R. A. Marcus, J. Chem. Phys. 81, 4494 (1984).

${ }^{7}$ R. A. Marcus, Rev. Mod. Phys. 65, 599 (1993).

${ }^{8}$ H. Bässler, Phys. Status Solidi B 175, 15 (1993).

${ }^{9}$ P. M. Borsenberger and D. S. Weiss, Organic Photoreceptors for Xerography (Dekker, New York, 1998).

${ }^{10}$ P. W. M. Blom and M. C. J. M. Vissenberg, Mater. Sci. Eng. 27, 53 (2000).

${ }^{11}$ V. I. Arkhipov, I. I. Fishchuk, A. Kadashchuk, and H. Bässler, in Semiconducting Polymers, edited by G. Hadziioannou and G. G. Malliaras (Wiley, Weinheim, Germany, 2007), Vol. 1, p. 275.

${ }^{12}$ V. Coropceanu, J. Cornil, D. A. da Silva Filho, Y. Olivier, R. Silbey, and J. L. Brédas, Chem. Rev. 107, 926 (2007).

${ }^{13}$ L. Sudha Devi, M. K. Al-Suti, C. Dosche, M. S. Khan, R. H. Friend, and A. Köhler, Phys. Rev. B 78, 045210 (2008).

${ }^{14} \mathrm{~S}$. T. Hoffmann, S. Athanasopoulos, D. Beljonne, H. Bässler, and A. Köhler, J. Phys. Chem. C 116, 16371 (2012).

${ }^{15}$ S. V. Novikov, D. H. Dunlap, V. M. Kenkre, P. E. Parris, and A. V. Vannikov, Phys. Rev. Lett. 81, 4472 (1998).

${ }^{16}$ W. F. Pasveer, J. Cottaar, C. Tanase, R. Coehoorn, P. A. Bobbert, P. W. M. Blom, D. M. de Leeuw, and M. A. J. Michels, Phys. Rev. Lett. 94, 206601 (2005).

${ }^{17}$ R. Coehoorn, W. F. Pasveer, P. A. Bobbert, and M. A. J. Michels, Phys. Rev. B 72, 155206 (2005).

${ }^{18}$ V. I. Arkhipov, P. Heremans, E. V. Emelianova, G. J. Adriaenssens, and H. Bässler, J. Phys.: Condens. Matter 14, 9899 (2002).

${ }^{19}$ I. I. Fishchuk, V. I. Arkhipov, A. Kadashchuk, P. Heremans, and H. Bässler, Phys. Rev. B 76, 045210 (2007).

${ }^{20}$ A. Miller and E. Abrahams, Phys. Rev. 120, 745 (1960).

${ }^{21}$ H. Bässler, P. M. Borsenberger, and R. J. Perry, J. Polym. Sci., Part B: Polym. Phys. 32, 1677 (1994).
${ }^{22}$ P. E. Parris, V. M. Kenkre, and D. H. Dunlap, Phys. Rev. Lett. 87, 126601 (2001).

${ }^{23}$ I. I. Fishchuk, A. Kadashchuk, H. Bässler, and S. Nešpưrek, Phys. Rev. B 67, 224303 (2003).

${ }^{24}$ J. Cottaar, L. J. A. Koster, R. Coehoorn, and P. A. Bobbert, Phys. Rev. Lett. 107, 136601 (2011).

${ }^{25}$ A. Köhler and H. Bässler, J. Mater. Chem. 21, 4003 (2011).

${ }^{26}$ S. T. Hoffmann, E. Scheler, J.-M. Koenen, M. Forster, U. Scherf, P. Strohriegl, H. Bässler, and A. Köhler, Phys. Rev. B 81, 165208 (2010).

${ }^{27}$ V. I. Arkhipov, E. V. Emelianova, H. Bässler, and A. Kadashchuk, Chem. Phys. 266, 97 (2001).

${ }^{28}$ D. Monroe, Phys. Rev. Lett. 54, 146 (1985).

${ }^{29}$ S. D. Baranovskii, H. Cordes, F. Hensel, and G. Leising, Phys. Rev. B 62, 7934 (2000).

${ }^{30}$ R. Schmechel, Phys. Rev. B 66, 235206 (2002).

${ }^{31}$ G. A. H. Wetzelaer, L. J. A. Koster, and P.W. M. Blom, Phys. Rev. Lett. 107, 066605 (2011).

${ }^{32}$ S. Kirkpatrick, Rev. Mod. Phys. 45, 574 (1973).

${ }^{33}$ V. Ambegaokar, B. I. Halperin, and J. S. Langer, Phys. Rev. B 4, 2612 (1971).

${ }^{34}$ J. Cottaar, R. Coehoorn, and P. A. Bobbert, Phys. Rev. B 85, 245205 (2012).

${ }^{35}$ J. O. Oelerich, D. Huemmer, M. Weseloh, and S. D. Baranovskii, Appl. Phys. Lett. 97, 143302 (2010).

${ }^{36}$ K. Kohary, H. Cordes, S. D. Baranovskii, P. Thomas, S. Yamasaki, F. Hensel, and J.-H. Wendorff, Phys. Rev. B 63, 094202 (2001)

${ }^{37}$ J. Cottaar and P. A. Bobbert, Phys. Rev. B 74, 115204 (2006).

${ }^{38}$ S. T. Hoffmann, H. Bässler, J.-M. Koenen, M. Forster, U. Scherf, E. Scheler, P. Strohriegl, and A. Köhler, Phys. Rev. B 81, 115103 (2010).

${ }^{39}$ S. T. Hoffmann, F. Jaiser, A. Hayer, H. Bässler, T. Unger, S. Athanasopoulos, D. Neher, and A. Köhler, J. Am. Chem. Soc. 135, 1772 (2013). 